\title{
A study on verification of sphygmomanometers.
}

\author{
Tavakoli Golpaygani Ali ${ }^{*}$, Keshavarz Mohammad Amin ${ }^{2}$, Dehghan Abnavi Jalil ${ }^{3}$ \\ ${ }^{1}$ Department of Biomedical Engineering, Standard Research Institute, Karaj, Iran \\ ${ }^{2}$ Department of Medical Care, Shiraz University of Medical Sciences, Shiraz, Iran \\ ${ }^{3}$ Persia Azma System Co., Shiraz, Iran
}

\begin{abstract}
Blood pressure measurement is an essential component of the general examination of every patient and the sphygmomanometer is one of the most commonly used diagnostic medical devices. The accuracy of sphygmomanometers depends strictly on performing the correct maintenance and calibration process of this equipment. The aim of this study was assessing the calibration condition of sphygmomanometers in use of primary care especially in physicians and general practitioner's office. The metrological reliability of more than two hundred manual sphygmomanometers in use in over 150 physician's offices and small clinics was evaluated. Quantitative analysis showed the amount of the obtained results in some equipment is critical and over the standard limitations. Primary care practitioners should have a system to ensure that their sphygmomanometers are recalibrated regularly according to the national or international standards. Using a mal-calibrated medical device has serious ethical, social and health implication to the society in general and to the misdiagnosed or mistreatment patient in particular. So, the obtained results show there is serious need for new and severe regulations on periodic performance verifications and medical equipment quality control program.
\end{abstract}

Keywords: Medical devices, Sphygmanometers, Verifications, Quality control.

Accepted on June 11, 2018

\section{Introduction}

Nowadays more than 10,000 different types of medical devices can be found in hospitals, in a wide variety with different physiological effects and measurements. Medical centers must ensure that their medical devices are safe, accurate and operating at the required level of performance $[1,2]$.

Uncontrolled high blood pressure greatly increases the risk of heart disease and stroke which are the first and third leading causes of death in developed countries. Because there are no symptoms, many people with hypertension do not know they have it. The blood pressure measurement is one of the commonly performed procedures in primary care stages by the physicians. The correct measurement of arterial blood pressure is essential for the effective primary prevention of cardiovascular diseases [3].

Accurate measurement of blood pressure requires the use of an accurate sphygmomanometer. A manual sphygmomanometer is a device used to measure blood pressure, and consists of an inflatable cuff, mercury manometer (or aneroid gauge) and inflation bulb and gauge. The inflatable cuff is used to restrict blood flow, and manometer is used to measure the pressure. Figure 1 shows some different kinds of sphygmomanometers $[1,2]$.

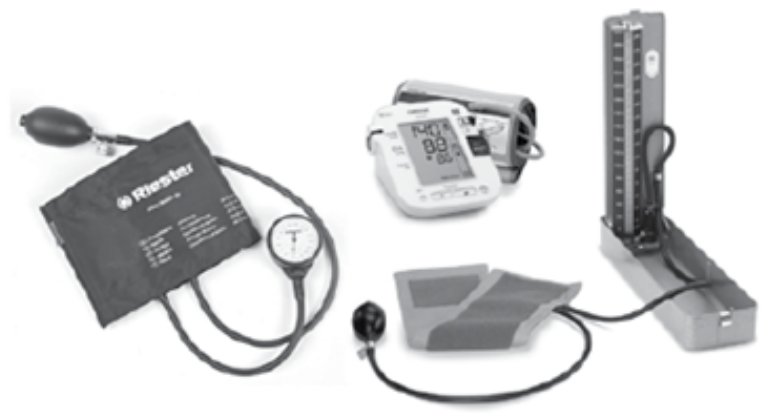

Figure 1. Different kinds of sphygmomanometers.

One of the most common errors in BP measurement is caused by using mal-calibrated sphygmomanometer and miscuffing. Inadequate sphygmomanometer maintenance and calibration is a cause for systematic error in BP measurements. Since few practices have a system which ensures that sphygmomanometers are accurate, it appears all patients are at risk of this kind of measurement error. Hence the need arises for proper calibration of these instruments as well as choosing the right cuff size for the right patient to minimize these errors [2]. 


\section{Materials and Methods}

The metrological reliability of more than two hundred manual sphygmomanometers in use in over 150 physicians' offices and small public and private clinics in one of the megacities of Iran according to international and national standards were evaluated. The calibration of electronic automated sphygmomanometers was not performed.

All sphygmomanometers, were tested and certified according to international standard (AAMI/ANSI SP10 and ISO 81060-1, 2) $[4,5]$. There are several parameters that impact the performance of a sphygmomanometer. Table 1 demonstrated some of these parameters.

Table 1. Performance parameters of manual sphygmomanometers.

\begin{tabular}{ll}
\hline Qualitative Tasks & Quantitative Tasks \\
\hline Tubes/Hoses/Bulb & Pressure accuracy \\
\hline Fitting/Connectors & Pressure leakage \\
\hline Gauge/Column & Zero pressure setting \\
\hline Cuffs/Labeling & Hysteresis response \\
\hline
\end{tabular}

After performing a visual check, the performance of the under test device is assessed with a Biomedical Analyzer (Prosim8). By a Y connector under test device was connected to analyzer, the maximum air pressure in evaluation process was increased to $250 \mathrm{mmHg}$. The pressure accuracy was evaluated in both of the static and dynamic conditions. Any difference in pressure between the standard and tested sphygmomanometers was noted for each piece of equipment.

\section{Results and Discussion}

Detection of hypertension and classification of an individual as being hypertensive or not is decided based on the measurement of blood pressure by a sphygmomanometer. However this measurement is not without its limitations in accuracy with observer faults as well as instrumental errors. It is evident that errors in blood pressure measurement can have important implications to the patients; a systematic error of even $5 \mathrm{mmHg}$ can double or halve the number of patients diagnosed with hypertension. For example, it is seen when a person with a real $90 \mathrm{mmHg}$ diastolic blood pressure is wrongly noted as 95 $\mathrm{mmHg}$ this person will be classified as a hypertensive person and put on treatment which he does not need in reality and will confer entirely unnecessary expenditures for people. In contrary, a systematic measurement error of $5 \mathrm{mmHg}$ downwards overlooks the need for treatment of a patient with a diastolic blood pressure of $95 \mathrm{mmHg}$. Ultimately, these errors in measurements could lead to increases in serious medical and social complications [6].

The mercury sphygmomanometers provide more accurate results than aneroid sphygmomanometers, but aneroid equipment is increasingly being used due to ease of handling and fewer environmental concerns. In addition to the observer bias in measurement of blood pressure, other important parameters are using a faulty and inaccurate sphygmomanometer and miscuffing. Most GPs and physicians use a regular-sized cuff to measure blood pressure in patients with different arm circumference, if the size of cuff is not adequate for patient's arm, this can cause under or overestimation of blood pressure up to $8 \mathrm{mmHg}$.

This study found that $78 \%$ of physicians and practitioners had never ( 3 y) calibrated their sphygmomanometers, in this way many of them did not known when their equipment had last been tested. More than one-third of practitioners had no programming and arrangement for periodic maintenance and calibration process until the need for repairmen in the future.

In more than two-thirds of tested sphygmomanometers no testing had taken place for at least a year. We found over $22.4 \%$ of sphygmomanometers had $4 \mathrm{mmHg}$ errors and more than it. Nearly $70 \%$ of under test equipment had degree of measurement error between -2.0 to $+2.0 \mathrm{mmHg}$, and $1.2 \%$ gave readings which were inaccurate by more than $6 \mathrm{mmHg}$. About 18 sphygmomanometers were in such a poor physical state, that they had air leakages in tubing, connector and fitting or dirt in the mercury, which suggested they should be withdrawn from service. There was no relationship between the age and accuracy of sphygmomanometers, with some new devices giving inaccurate readings.

In some of the sphygmomanometers the zero point not be adjusted and the practitioners had no concern about this matter and did not consider this bias in their measurements.

\section{Conclusion}

Blood pressure is simple but vital physiological parameters to evaluate performance of cardiovascular organ. A sphygmomanometer is a device used to measure blood pressure, and accurate measurement of blood pressure requires the use of an accurate sphygmomanometer. Inadequate sphygmomanometer maintenance and calibration is a cause for systematic error in BP measurements. Similarly errors in BP measurement arise when the cuff size is too small relative to the patients arm circumference or vice versa.

Hence sphygmomanometers need to be calibrated at regular intervals to prevent them from giving erroneous measurements as well as choosing the right cuff size for the right patient arm to minimize these errors. This periodic evaluation should be done at an interval not greater than 12 months and rechecked at an interval of 6 months for optimum readings.

Primary care practitioners have a clinical and ethical responsibility to ensure their equipment are appropriately serviced and maintained according to a predefined program. As a minimum, all physicians and GPs should have a system to ensure that their sphygmomanometers are recalibrated regularly according to the national or international standards.

Using a mal-calibrated medical device has serious ethical, social and health implications to the society in general and to the misdiagnosed or mistreatment patient in particular. 


\section{References}

1. Khandpour RS. Handbook of biomedical instrumentation. McGraw Hill 2014.

2. Tavakoli Golpaygani A. Why should we have a periodic safety and performance program for medical devices? J Biomed Phys Eng 2017.

3. National Heart Foundation of Australia. Guideline for the diagnosis and management of hypertension in adults. National Heart Foundation of Australia 2016.

4. ISO 81060-1:2007. Non-invasive sphygmomanometersPart 1: Requirements and test methods for non-automated measurement type. Int Organization Standardization 2007.

5. ISO 81060-2:2013. Non-invasive sphygmomanometersPart 2: Clinical investigation of automated measurement type. Int Organization Standardization 2013.
6. Turner MJ, Kam PC, Baker AB. Metrology in medicine. Department of Anesthetics, University of Sydney 2004.

\section{*Correspondence to}

Tavakoli Golpaygani Ali

Department of Biomedical Engineering

Standard Research Institute

Karaj

Iran 